\title{
Simplest laboratory design of differential thermal analyzer
}

\author{
Somnath Danayak ${ }^{1}$ and Kamal K. Kar ${ }^{2,3 *}$ \\ ${ }^{1}$ Materials Instructional Laboratory, ${ }^{2}$ Advanced Nanoengineering Materials laboratory, \\ ${ }^{3}$ Materials Science Programme, Indian Institute of Technology Kanpur, Kanpur-208016, India \\ *Email: kamalkk@iitk.ac.in
}

Materials undergo structural and compositional changes due to phase transition, melting, crystallization, decomposition etc. These changes can be studied using DTA. It consists of measuring differential temperature $\Delta \mathrm{T}$ (called signal) between sample and a standard material when both are heated or cooled at a constant rate. As the sample undergoes a phase transition it absorbs or emits heat and the sample becomes cooler or warmer than the standard material. It give rise to DTA peaks [1].

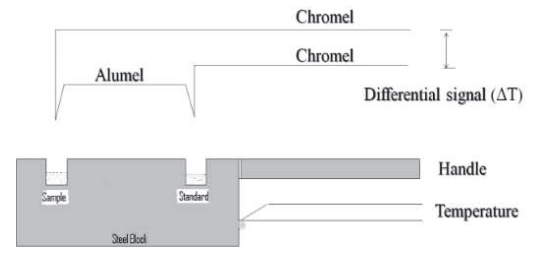

Figure 1: Thermocouple connected in differential mode and DTA pans drilled in steel block

Figure 1 shows the designing technique used to prepare the instrument. For placing test sample and standard material two cups were drilled in a same steel block to maintain uniform temperature throughout. It will minimize undesired signal drift. Two thermocouples connected in differential manner is the heart of the instrument. Here two Chromel-Alumel thermocouples are connected to produce differential temperature $\left(\Delta \mathrm{T}=\mathrm{T}_{1}-\mathrm{T}_{2}\right)$ or DTA signal. These are fitted in a movable arm such that the tip of each of these TC's can move into two cups provided in the steel block (Figure 2).

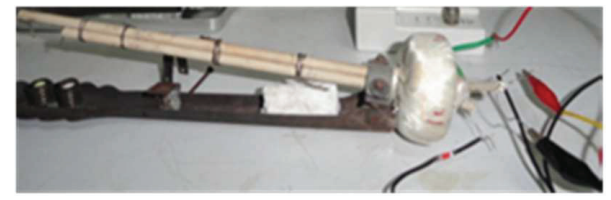

Figure 2: Sample holder: Tips of the differential thermocouples are arranged to move into DTA pans through movable arm

A homemade Kanthal wound tubular furnace was used for heating purposes. DTA signal and temperature sensor outputs were connected to temperature module of National Instruments data acquisition system, which provided a DTA plot directly on computer screen (Figure 3).

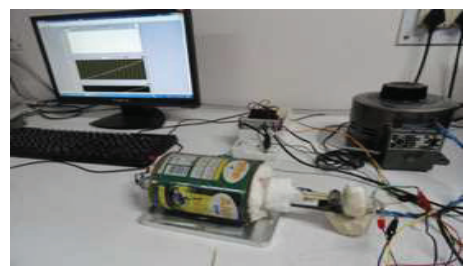

Figure 3: Test setup containing DTA and data acquisition system
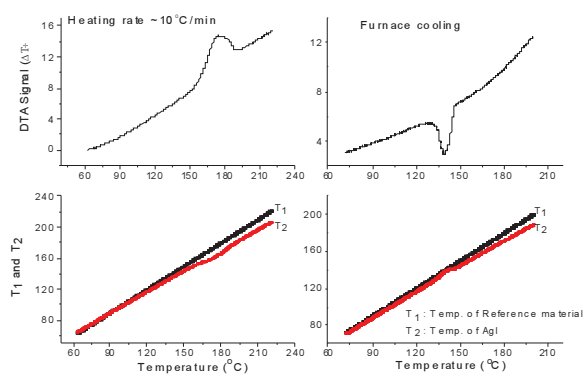

Figure 4: Differential temperature $(\Delta \mathrm{T})$ versus temperature plot showing phase transition of $\mathrm{AgI}$ for heating and cooling cycle

Here we studied the $\beta$ to $\alpha$ phase transition temperature of $\mathrm{AgI}$ and observed corresponding endothermic and exothermic peaks for heating and cooling cycle respectively. It was found very close to literature value $\left(148^{\circ} \mathrm{C}\right)$ as shown in Figure 4.

The result was found quite correct. Associated energy can also be found by measuring the area under peak once calibrated with a calorimeter. Signal drift, as shown can be further minimized by using a precise furnace. Multimeter along with interfacing adapter can also be used instead of data acquisition system.

\section{Reference}

1. E.C. Subbarao, L.K. Singhal, D. Chakravorty and Masshal F. Merriam, Experiments in Materials Science. 